\title{
Interstellar Grain Flow through the Solar Wind Cavity around 1992
}

\author{
Bo A. S. Gustafson and Susan M. Lederer \\ Department of Astronomy, 211 SSRB, University of Florida, \\ Gainesville, Fl 32611, U.S.A.
}

\section{Introduction}

The local cloud in which the Sun is embedded participates in a general flow from the direction of the Sco-Cen association (Weaver, 1979) while the Sun is moving in the direction of Hercules. The relative motion is well determined by the neutral gas flow (Cox and Reynolds, 1987). A prominent cone of gravitationally focused neutral helium indicates the downwind direction to within $\pm 3^{\circ}$. The average flow speed outside the solar system, $26 \mathrm{~km} / \mathrm{s}$, is determined to within \pm 1 $\mathrm{km} / \mathrm{s}$. Measurements from the Ulysses spacecraft (Witte et al., 1993) confirmed this flow. Since interstellar matter contains dust as well as gas, efforts have been made to detect the dust flow through the solar system. However, until recently these efforts have been unsuccessful and modeling of the flow has been controversial.

Several researchers have suggested that the Lorentz force prevents interstellar grains from penetrating to depths in the solar wind cavity where spacecrafts could detect them. However, numerical simulations by Gustafsori and Misconi (1979) and by Grün et al. (1994) showed that interstellar grains can reach regions accessible to spacecraft. Measurements from the Ulysses and Galileo deep-space probes appear to have confirmed these predictions (Grün et al., 1994).

\section{The Modeling}

We adopt Parker's model of the solar wind and its solar magnetic cycle dependence as described by Gustafson (1994). This leads to a rather rugged qualitative modeling of the Lorentz force. Specifically, the solar wind speed has a negligible effect (Gustafson, 1994). Other model parameters are also relatively unimportant, or affect mostly the average magnitude of the Lorentz force per unit mass, thus masquerading as a change in the charge to mass ratio. Because the grain charge and to some extent the mass distribution are uncertain, we must consider a range of charge to mass ratios anyway. Gravity and radiation pressure on classical size interstellar grains are expected to nearly cancel each other (Gustafson, 1994). Thus, the second order gravity and radiation pressure force can be ignored. This decreases the number of parameters and highlights the charge to mass ratio dependency.

All grains are assumed to be at rest with respect to the local interstellar gas as they enter the solar wind cavity. The adopted incident direction of $7^{\circ}$ north latitude, indicated by many measurements, lies approximately one standard deviation from the Ulysses data that favor $2.5^{\circ}$ north (Witte et al., 1993). 


\section{Results}

Interstellar grains entering the solar wind cavity $100 \mathrm{AU}$ ahead of the Sun and moving at $26 \mathrm{~km} / \mathrm{s}(\approx 5.5 \mathrm{AU} / \mathrm{yr})$ typically experience more than one 11 year half cycle of the magnetic field configuration. The Lorentz force accelerates grains on either side of the solar equator toward each other when the solar magnetic field points north, and away from the equator when the field points south (Gustafson, 1994). The drift velocity depends on the integrated acceleration as grains move both in the focusing field and in the defocusing field. The solar wind sector structure thus causes a relatively benign deflection at low latitudes.

The dominating magnetic field in the 1980s focused the grain flow along the equatorial plane, but the direction of drift may lag the direction of acceleration by as much as half a solar magnetic cycle (i.e., an 11 year lag). Grains that come close to the Sun, where the solar wind is dense, are strongly accelerated in the defocusing magnetic field configuration, which has developed since the last solar maximum. The Lorentz force can even reverse the flow direction of some grains. Such grains may have been detected from Ulysses (Grün, private communication).

Figure 1a shows that the regular spacing of grains with a charge of 0.6 $\mathrm{C} / \mathrm{kg}$ (see Table 1) is maintained over much of the volume but a wedge shaped concentration develops downstream from the Sun. The decreasing deflection angle toward the equatorial plane with decreasing heliocentric latitude results in intersecting trajectories at shallow angles and causes the wedge shape. The concentration strengthens and migrates upstream as the charge to mass ratio increases. A higher latitude concentration also emerges when the charge increases to $3.7 \mathrm{C} / \mathrm{kg}$ (Figure $1 \mathrm{~b}$ ). The high latitude concentration has a circular shape in this projection and develops as the Lorentz force decelerates grains at high heliocentric latitudes. The structure $>40$ AU downstream is a remnant of the wedge shaped concentration that developed near the Sun a full 22 year magnetic cycle earlier. Figure 1c shows how the extent of both concentrations grow as the charge increases to $8.3 \mathrm{C} / \mathrm{kg}$. Increasing the charge to $530 \mathrm{C} / \mathrm{kg}$ extends the high latitude phenomenon to low latitudes. The grains couple tightly to the solar wind and are swept away in a process mimicking ion interactions at the edge of the solar wind cavity. The translation of charge to mass ratio to radius and mass of spherical grains is given in Table 1.

Table 1. Charge to mass ratio (Q/M) and corresponding particle parameters assuming spherical grain shapes

\begin{tabular}{|c|c|c|c|c|c|c|c|c|}
\hline $\mathbf{Q} / \mathbf{M}$ & \multicolumn{4}{|c|}{+5 Volt } & \multicolumn{4}{|c|}{+20 Volt } \\
\hline & $\rho=2$. & $\mathrm{g} / \mathrm{cm}^{3}$ & $\overline{\rho=1}$ & $\mathrm{~cm}^{3}$ & $\overline{\rho=2 . !}$ & $/ \mathrm{cm}^{3}$ & $\overline{\rho=1}$ & $/ \mathrm{cm}^{3}$ \\
\hline $\mathrm{C} / \mathrm{kg}$ & $\begin{array}{l}\text { Radius } \\
\mu \mathrm{m}\end{array}$ & $\begin{array}{l}\text { Mass } \\
\mathrm{g}\end{array}$ & $\begin{array}{l}\text { Radius } \\
\mu \mathrm{m}\end{array}$ & $\begin{array}{c}\text { Mass } \\
\mathrm{g}\end{array}$ & $\begin{array}{l}\text { Radius } \\
\mu \mathrm{m}\end{array}$ & $\begin{array}{c}\text { Mass } \\
\mathrm{g}\end{array}$ & $\begin{array}{l}\text { Radius } \\
\mu \mathrm{m}\end{array}$ & $\begin{array}{l}\text { Mass } \\
\mathrm{g}\end{array}$ \\
\hline 0.6 & 0.30 & $2.8 \mathrm{E}-13$ & 0.47 & $4.3 \mathrm{E}-13$ & 0.60 & $2.2 \overline{\mathrm{E}}-12$ & 0.95 & $3.6 \mathrm{E}-12$ \\
\hline 3.7 & 0.12 & $1.8 \mathrm{E}-14$ & 0.19 & $2.9 \mathrm{E}-14$ & 0.24 & $1.5 \mathrm{E}-13$ & 0.38 & $2.3 \mathrm{E}-13$ \\
\hline 8.3 & 0.08 & $5.4 \mathrm{E}-15$ & 0.12 & $8.2 \mathrm{E}-15$ & 0.16 & $4.3 \mathrm{E}-14$ & 0.25 & $6.8 \mathrm{E}-14$ \\
\hline 530.0 & 0.01 & 1.0E- 17 & 0.02 & $1.7 \mathrm{E}-17$ & 0.02 & $8.4 \mathrm{E}-17$ & 0.03 & $1.4 \mathrm{E}-16$ \\
\hline
\end{tabular}

Figure $2 \mathrm{a}$ is a $\pm 1 \mathrm{AU}$ thick cut along the solar equatorial plane through a cloud of $3.7 \mathrm{C} / \mathrm{kg}$ grains. The figure shows that the grain concentration caused 

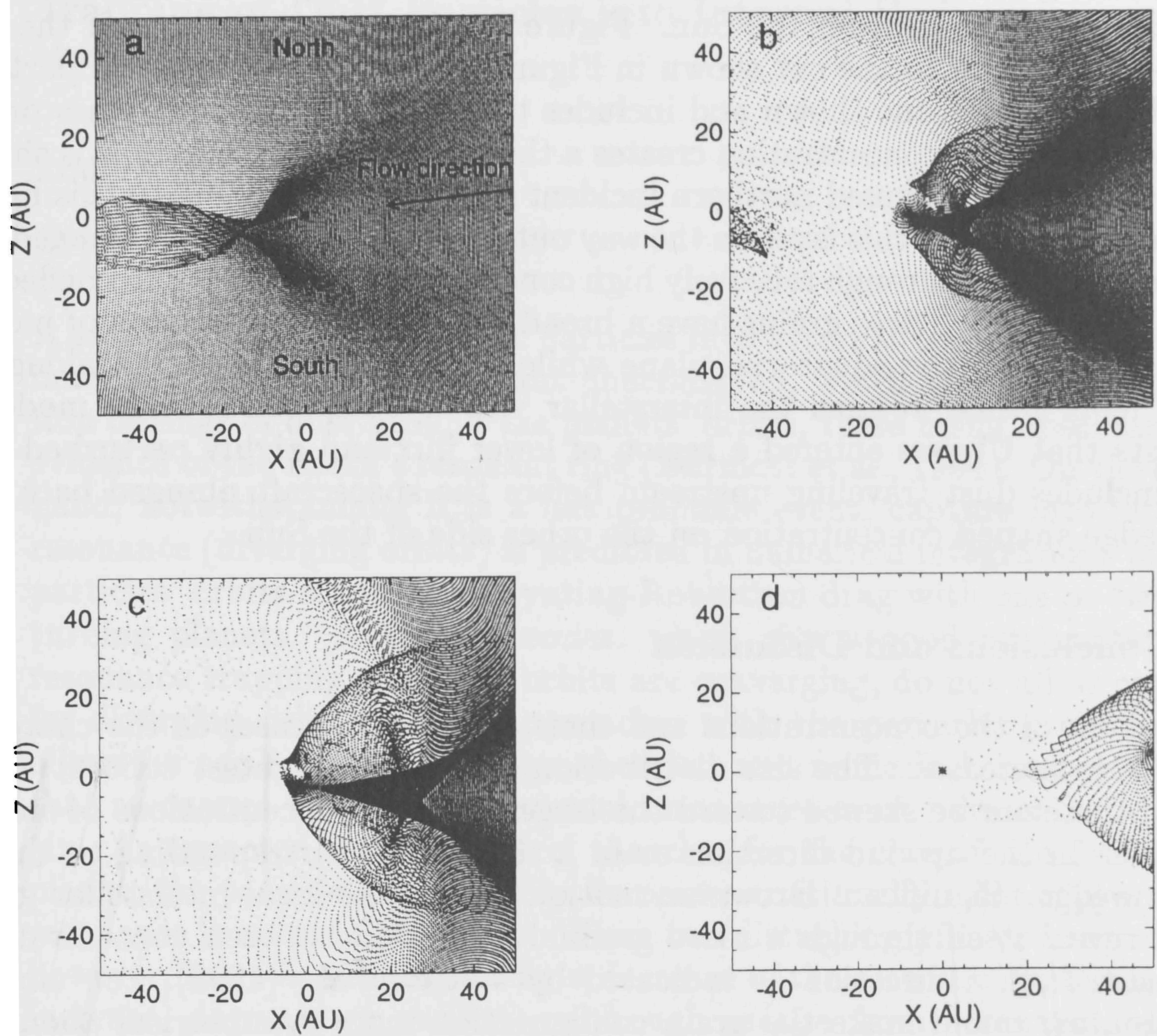

Figure 1. Snapshot of particle locations in 1992. The 1 AU thick cut is perpendicular to the ecliptic and includes the Sun and the incident flow direction. The charge to mass ratio dependence is illustrated using $0.6 \mathrm{C} / \mathrm{kg}$ test grains in (a), $3.7 \mathrm{C} / \mathrm{kg}$ in (b), $8.3 \mathrm{C} / \mathrm{kg}$ in (c), and 530 $\mathrm{C} / \mathrm{kg}$ in (d).
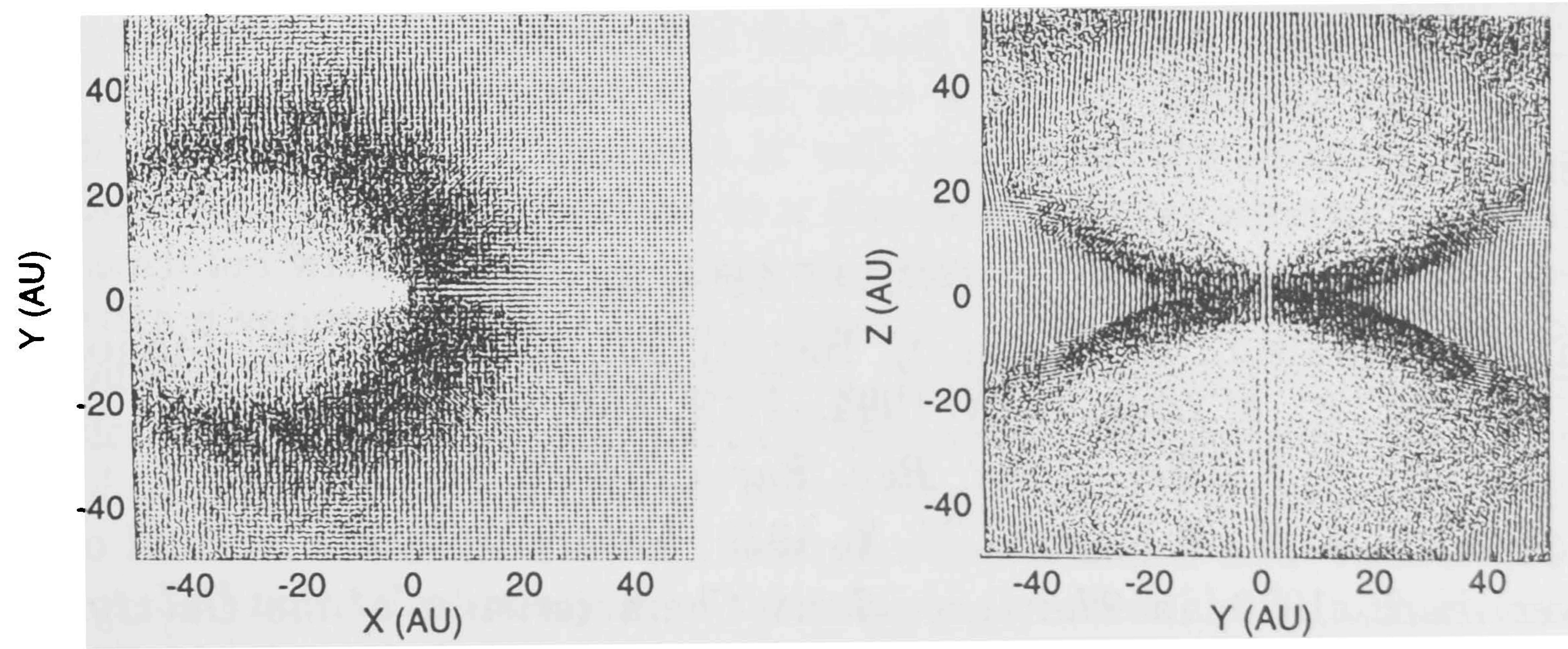

Figure 2. Location of $3.7 \mathrm{C} / \mathrm{kg}$ particles in 1992. The $2 \mathrm{AU}$ thick cut in (a) is along the solar equatorial plane. (b) shows the perpendicular cut through the Sun orthogonal to the cut shown in Figure 1. 
by the wedge shaped structure is drawn out in an ellipse. A depleted zone extends downstream from the Sun. Figure 2b shows a slice through the Sun and perpendicular to the cut shown in Figure 1. The slice includes all particles $\pm 1 \mathrm{AU}$ from the plane shown and includes the trajectory of the Ulysses probe. The wedge shaped concentration creates a thin layer near the Sun that is shifted south due to the assumed northern incident direction. According to this figure, Ulysses flew north of this layer on the way out to Jupiter. The probe plunged into the sheet, passing through relatively high concentrations, following the deflection south by Jupiter. These grains have a broad spread in their direction of motion perpendicular to the equatorial plane while the velocity component along the plane more nearly retains the interstellar velocity dispersion. The modeling suggests that Ulysses entered a region of lower flux and highly perturbed dust that includes dust traveling upstream before the spacecraft plunged back into the wedge shaped concentration on the other side of the Sun.

\section{Conclusions and Discussion}

The extent of the concentrations and their strength increases as the charge to mass ratio increases. The size distribution outside the tightest concentrations should therefore be skewed toward the larger grains. Concentrations of smaller grains lie in the upwind direction or in a thin sheet, corresponding to the tip of the wedge. Significant Brownian motion among the local interstellar grains might reveal itself through a more gradual transition between concentrations. Incidence from a direction as indicated by Witte et al. (1993), i.e., close to the equator, would make the grain configuration more symmetrical about the equatorial plane but otherwise has a negligible effect.

Further modeling has shown that the radiation pressure force can create an empty wake behind the Sun. A gravitational enhancement over the interstellar number density occurs only for larger grains. The wedge shaped concentration slips past the Sun and splits into northern and southern components as the solar magnetic cycle progresses.

Acknowledgments. This work was supported in part by NASA grant NAGW-2775.

\section{References}

Cox, D. P., \& Reynolds, R. J. 1987, ARA $\& A$, 25, 303

Grün, E., Gustafson, B., Mann, I.. 'Baguhl, M., Morfill, G. E., Staubach, P., Taylor, A., \& Zook, H. A. 1994, A\& A, 286, 915

Gustafson, B. A. S. 1994, Annu. Rev. Earth Planet. Sci., 22, 553

Gustafson, B. A. S., \& Misconi, N. Y 1979, Nature, 282, 276

Weaver, H. F 1979, in The Large-Scale Characteristics of the Galaxy, W. B. Burton, Dordrecht: Reidel, 295

Witte, M., Rosenbauer, H., Banaszkiewicz, M., \& Fahr, H. 1993, Advances in Space Res., 13(6), 121 